\title{
Autism, Eye-Tracking, Entropy
}

\author{
Frederick Shic, Katarzyna Chawarska, Jessica Bradshaw, Brian Scassellati \\ Yale University \\ New Haven, CT, USA
}

\begin{abstract}
Using eye-tracking, we examine the scanning patterns of 2 year old and 4 year old toddlers with and without autism spectrum disorder as they view static images of faces. We use several measures, such as the entropy of scanning patterns, in order to characterize the differences in attention towards faces by these children. We find a differential pattern of both fine attention (towards specific regions of the face) as well as gross attention (looking at the faces at all) which seem to suggest different developmental trajectories for the two groups of children. We discuss the implications of these trends and, additionally, discuss current methods in eye-tracking and the development of simple, effective, and robust measures and methodology for evaluating scanning patterns.
\end{abstract}

Keywords- autism; eye-tracking; entropy; Markov; faces

\section{INTRODUCTION}

Autism is a pervasive developmental disorder marked by social and communicative deficits as well as stereotyped and repetitive behaviors [1]. While communicative deficits and repetitive behaviors are found in many developmental disorders, the seriousness and specificity of social deficits are a particular hallmark of autism. How these social deficits arise, and how early deficits impact the development of a child with autism is a crucial and difficult question. In this paper we investigate how children with autism examine the most social form known to us: the human face.

It has long been known that individuals with autism exhibit deficits and abnormalities in recognizing or making complex judgments about faces (e.g. [2,3]), though most of these studies are conducted in adults or higher functioning adolescents with autism. For example, Klin et al. [4] showed that adolescents with autism, during a free-viewing of dynamic naturalistic social scenes, tended to focus more upon the mouth, body, and objects in the scene, when compared to matched controls. Similarly, Pelphrey et al. [5] found that the scanning patterns of individuals with autism seemed more erratic and tended towards less informative regions of a static face, as compared to typical controls.

Our work asks: how do the face scanning strategies of children with autism change in early childhood? In other words, this work seeks to uncover trends that can characterize the developmental progression of the disorder. To accomplish this, we build an analytical methodology for eye-tracking using measures and tools requiring little computational muscle, yet which are still quite powerful and penetrative.

This project was supported by NIH Research Grant U54 MH66494 funded by the NIMH, NIDCD, NIEHS, NICHD, and NINDS; NSF CAREER award (\#0238334) and NSF award \#0534610; a software grant from QNX Software Systems Ltd.; and support from the Sloan Foundation. Generous research and training grants were also provided by Autism Speaks and NAAR.
Some of the tools that we will use are simple measures taken from information theory. Specifically, we employ entropy measures and Markov chains in order to cut complex gaze trajectories into elementary statistics. These methods can directly remark on the randomness or stereotypical nature of scanning patterns. Similarly, it is our hope that by applying our techniques to the scanning of faces, we can also comment on biases in autism towards different areas of the face which deviate from typical development. In our discussion, we will address both social and developmental aspects of our findings.

\section{SUBJECTS AND EXPERIMENTAL METHODS}

\section{A. Subjects}

51 children participated in this study, divided between two diagnostic categories and two age groups. The younger age group consisted of 12 toddlers with autism spectrum disorder (ASD) (mean age 25.6(5.6) months) and 13 typically developing (TD) toddlers (mean age 25.1(6.0) months). The older age group consisted of 13 children with ASD (mean age 43.6(5.4) months) and 13 TD children (mean age 45.0(4.3) months). Diagnosis of ASD was obtained at the time of testing through standardized assessment instruments [6,7] and expert clinical observations. For further details and a discussion regarding the stability of early diagnosis, see [8].

\section{B. Experimental Protocol and Data Reduction}

Children were presented with 6 color images of faces [9] (Fig. 1a) centered at a distance of $75 \mathrm{~cm}$ from the centerline of the children's eyes. Experiments for Age Group 1 (the younger group) were conducted on a 20 " widescreen LCD monitor, such that the stimulus (including grey background) measured $12.8^{\circ}$ wide by $17.6^{\circ}$ tall. Experiments for Age Group 2 (the older group) were conducted on a 24 " widescreen monitor, such that that the stimulus measured $15^{\circ}$ wide by $21^{\circ}$ tall. We will later return to these differences.
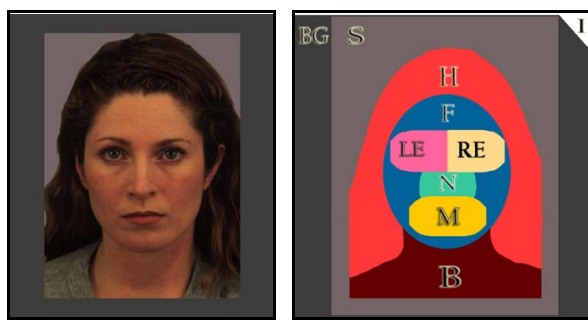

Figure 1. Stimulus image (left) and regions-of-interests (ROIs, right). The abbreviations for regions are: I(invalid data), BG (background), S(background of stimulus), $\mathrm{H}$ (hair), $\mathrm{F}$ (face), $\mathrm{LE}$ (left eye), RE(right eye), N(nose), M(mouth), B(body).

Stimuli were presented until the child had examined the image for a total of 10 seconds (as determined on-line by a 
trained experimenter) as part of a Visual Paired Comparison protocol (VPC) [10]. However, in this study we only examined the first 10 seconds of eye-tracking data (whether or not the child was fully attentive) in order to collect comparable information regarding inattention to stimuli. We also did not examine the recognition phase of the VPC.

Quality measures included checks on the consistency of eye-tracking calibration and a requirement that the 10 seconds of looking be acquired before a cutoff of 20 seconds. Only children who passed quality tests for at least half of the stimuli presentations were retained in this study. The 51 subjects of this study represent those children who met all data quality criteria. Results in this paper are presented at the subject level, with each subject's measures averaged over valid trails.

Gaze patterns were recorded with a SensoMotoric Instruments IView X RED table-mounted dark-pupil eyetracker operating at $60 \mathrm{~Hz}$. The tracking error reported by the manufacturer was less than $0.5^{\circ}$. Post processing was conducted through custom software written in Matlab and Perl and included standard automated recalibration.

\section{DATA ANALYSIS}

Face stimuli were broken into seven basic regions: the left eye, right eye, mouth, facial areas (face skin areas including the nose), outer features (hair and body), background, and nonstimulus (including blinks and periods where the child looked away from the eye-tracker) (Fig. 1b). These regions can be grouped in various ways in order to examine specific scanning interactions. However, even for our relatively small number of regions there were over a hundred possible groupings. For comparing two non-overlapping groups there were almost two thousand possible comparisons!

In order to manage the combinatorial explosion of comparisons we conducted a progressive regional analysis of the children's scanning patterns. This progressive analysis started at the highest level, including all possible regions, and gradually zoomed in on more information-dense facial regions. Three levels of analysis were employed. The top level (Level 3) began with an examination of the gross characteristics of attention, comparing scanning away from the main stimulus (non-stimulus regions: invalid data, stimulus background, screen background) with attention towards the stimulus (stimulus regions: eyes, mouth, skin areas, nose, hair, body). This analysis was followed by a mid-level of analysis which tapped information extraction from faces (Level 2), comparing the scanning of information-poor regions of the face (non-key regions: skin areas, nose, hair, body) with information-rich features (key regions: eyes, mouth). The final, and lowest, level of analysis focused on the canonical face processing circuit (Level 1) between the eyes (eye regions: left eye, right eye) and the mouth region.

\section{A. Static Time Analysis}

Measures for static analysis included the time spent in each region. Note that by "static" we do not mean to imply that the behavior of the scanning patterns, or their distributions, are time invariant, but rather that the statistics used do not carry information regarding transitions or dynamic behavior.

\section{B. Dynamic Time Analysis}

Measures for dynamic analysis included the number of transitions between outer (less informative) and inner (more informative) regions. We also considered the entropy $H$ (in base 2, i.e. in "bits" of information) of transition ratios in the three-stage functional circuit spanned by the outer area and the two subregions of the inner region:

$$
H(R)=-\sum_{r_{i} \in R} p\left(r_{i}\right) \log _{2} p\left(r_{i}\right)
$$

where $R$ is set of transitions under consideration and $p\left(r_{i}\right)$ is the ratio (probability) of looking at a particular transition $r_{i}$ belonging to $R$ [11]. We did not examine the two-stage outerinner circuit because in this case entropy provides the same information as a ratio. Typically, entropy is associated with randomness. However, in this context, entropy reflects a more even distribution of transitions between different regions. It is thus more closely aligned with a preference for exploration.

We also conducted a Markov chain entropy analysis of the scanning patterns of the three-stage outer-inner-subregions circuit. For each trial, we computed an approximate Markov matrix for that trial through sampling. We then characterized the entropy of the matrix. Given a $k$ th order Markov matrix $M$ with transition probabilities $m_{X i}$ where

$$
m_{X i}=p\left(X_{n+1}=r_{i} \mid X\right)
$$

and where $X$ is a $k$ th order history of past states [11], e.g. $X=$ $\left\{X_{n}=\right.$ eyes, $X_{n-1}=$ mouth $, \ldots, X_{k-1}=$ mouth $\}$ we can compute the entropy $H(X)$ as:

$$
H(X)=-\sum_{r_{i} \in R} m_{X i} \log _{2} m_{X i}
$$

and the entropy of matrix $M, H(M)$, as:

$$
H(M)=\sum_{X \in \mathbf{X}} p(X) H(X)
$$

with $\mathbf{X}$ the set of all possible histories. We do not model self transitions because they dominate in a frame by frame analysis, confounding switching rates with timing. Technically, the model we consider is a semi-Markov chain ignoring dwell. For brevity, we refer to these semi-Markov models as Markov. Similarly, the entropy above is the conditional entropy (dependent on history), which we simply refer to as entropy.

Note also that it is not possible to move between nonadjacent regions without crossing the interim area. For example, if we wish to scan between the eyes and the mouth, we must pass through the face. We have considered both raw unadulterated streams as well as streams with transitions lasting $50 \mathrm{~ms}$ or less removed. Transition counts and transition ratio entropies are transient-removed since they are easier to interpret when saccade effects are mitigated. Markov matrix results are raw since we can directly model transitional behavior by increasing model order. The process of transient removal did not alter our general pattern of results.

The information contained by transition rates, entropy of transition ratios, and entropy of the Markov matrix are complementary. Transition rates give an overview of how often movement is occurring between regions. The entropy of transition ratios marks how skewed the distribution of 
transitions are. The Markov matrix is a fine level frame-toframe predictive model of scanning. Note that the transition ratio entropy does not account for directional asymmetries whereas the Markov entropy does. For example, a clockwise pattern left-eye, right-eye, mouth back to left-eye might have a high transition ratio entropy, since transitions occur at equal frequency, but a 1st order Markov entropy would show that the pattern is essentially deterministic (zero entropy).

\section{RESUlTS}

In the interests of space, we have summarized the results of static analyses in Table 1 (time), the results of dynamic transition analyses in Tables 2 (counts) and 3 (entropy), and the results of Markov model analyses in Table 4. The reported results are based on multiple analyses of variance (MANOVA) with between-subject factors age and diagnosis.

TABLE I

Static Analysis: Time Spent in Region (ms)

\begin{tabular}{ccccc}
\hline \hline Region & \multicolumn{2}{c}{ age group 1 } & \multicolumn{2}{c}{ age group 2 } \\
\hline & ASD & TD & ASD & TD \\
NonStim & $3828(600)$ & $4612(666)$ & $5539(535)$ & $2429(220)$ \\
Stim & $6172(600)$ & $5388(666)$ & $4461(535)$ & $7571(220)$ \\
NonKey & $2150(264)$ & $1803(269)$ & $1628(233)$ & $2575(240)$ \\
Key & $4022(466)$ & $3586(473)$ & $2834(406)$ & $4995(294)$ \\
Mouth & $618(150)$ & $1151(328)$ & $535(110)$ & $1589(303)$ \\
Eyes & $3404(483)$ & $2434(539)$ & $2299(348)$ & $3406(315)$
\end{tabular}

TABLE II

Dynamic Analysis: Number of Transitions (count)

\begin{tabular}{ccccc}
\hline \hline Transition Regions & \multicolumn{2}{c}{ age group 1 } & \multicolumn{2}{c}{ age group 2 } \\
\hline & ASD & TD & ASD & TD \\
NonStim - Stim & $5.14(.54)$ & $5.60(.62)$ & $6.84(.64)$ & $5.81(.93)$ \\
NonStim - NonKey & $2.22(.36)$ & $2.08(.40)$ & $3.11(.32)$ & $2.34(.39)$ \\
NonStim - Key & $2.92(.35)$ & $3.52(.46)$ & $3.73(.50)$ & $3.47(.57)$ \\
NonKey - Key & $4.36(.59)$ & $3.80(.56)$ & $3.20(.47)$ & $5.79(.52)$ \\
NonKey - Eyes & $3.40(.50)$ & $2.43(.52)$ & $2.42(.46)$ & $4.05(.42)$ \\
NonKey - Mouth & $.96(.29)$ & $1.37(.43)$ & $.78(.21)$ & $1.74(.37)$ \\
Eyes - Mouth & $.88(.18)$ & $.76(.17)$ & $.89(.31)$ & $1.91(.35)$ \\
L.Eye-R.Eye & $1.46(.38)$ & $1.46(.43)$ & $1.28(.38)$ & $1.55(.32)$
\end{tabular}

TABLE III

Dynamic Analysis: Entropy of 3-stage Level Circuit (bits)

\begin{tabular}{ccccc}
\hline \hline Transition & \multicolumn{2}{c}{ age group 1 } & \multicolumn{2}{c}{ age group 2 } \\
\hline Level 3 & ASD & TD & ASD & TD \\
Level 2 & $1.39(.05)$ & $1.42(.04)$ & $1.50(.02)$ & $1.39(.05)$ \\
Level 1 & $.04(.16)$ & $1.06(.12)$ & $1.06(.13)$ & $1.29(.08)$ \\
& $.88(.14)$ & $.76(.16)$ & $.68(.19)$ & $1.22(.07)$
\end{tabular}

TABLE IV

Dynamic Analysis: Markov Chain Entropy (bits)

\begin{tabular}{cccccc}
\hline \hline \multicolumn{2}{c}{ Level Order } & \multicolumn{2}{c}{ age group 1 } & \multicolumn{2}{c}{ age group 2} \\
\hline & & ASD & TD & ASD & TD \\
3 & 0 & $1.480(.013)$ & $1.437(.032)$ & $1.522(.010)$ & $1.428(.025)$ \\
3 & 1 & $0.721(.032)$ & $0.651(.041)$ & $0.810(.022)$ & $0.607(.052)$ \\
3 & 2 & $0.578(.034)$ & $0.546(.041)$ & $0.678(.028)$ & $0.523(.043)$ \\
2 & 0 & $1.314(.029)$ & $1.246(.052)$ & $1.292(.028)$ & $1.367(.022)$ \\
2 & 1 & $0.414(.041)$ & $0.374(.050)$ & $0.424(.030)$ & $0.519(.031)$ \\
2 & 2 & $0.247(.041)$ & $0.216(.036)$ & $0.244(.030)$ & $0.367(.032)$ \\
1 & 0 & $1.272(.041)$ & $1.185(.074)$ & $1.265(.065)$ & $1.391(.025)$ \\
1 & 1 & $0.166(.043)$ & $0.094(.029)$ & $0.167(.029)$ & $0.257(.030)$ \\
1 & 2 & $0.064(.017)$ & $0.026(.012)$ & $0.045(.015)$ & $0.116(.018)$
\end{tabular}

\section{A. Level 3 (Top Level): Attention and Motivation}

Here we consider the functional circuit consisting of nonstimulus regions and stimulus regions. The stimulus region is further broken apart into key and non-key stimulus features.

A 2 (age) x 2 (diagnosis) analysis of looking time at the non-stimulus area indicated a significant effect of diagnosis $(\mathrm{F}(1,50)=4.8, \mathrm{p}<.05)$ and an age $\mathrm{x}$ diagnosis interaction, $(\mathrm{F}=13.4, \mathrm{p}<.001)$ (see Fig. 2a). Older TD children looked more at the stimulus than the younger group, $(\mathrm{F}=9.7, \mathrm{p}<.01)$, but in ASD the pattern was reversed, with older children looking less at the stimulus than younger ones $(\mathrm{F}=4.6, \mathrm{p}<.05)$.
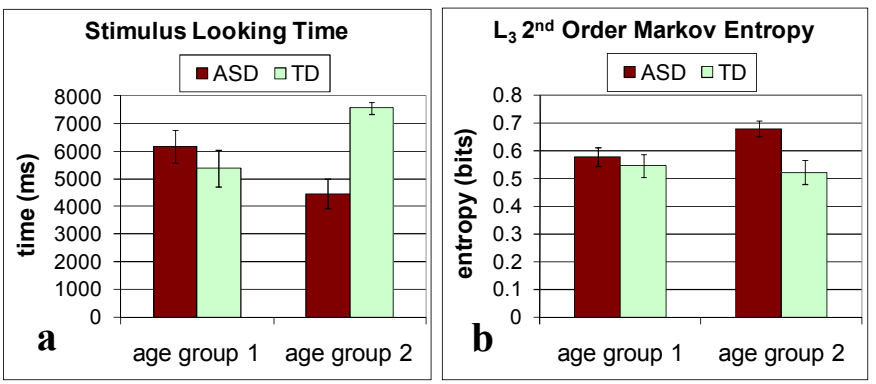

Figure 2. Measures from Level 3 circuit: (a) total average stimulus looking time (b) Markov matrix entropy of stimulus - non-key - key circuit. Error bars are standard error.

There were no significant effects of age and diagnosis on the number of transitions between non-stimulus and stimulus, which was surprising, given the discrepancy noted for times (see Table 2). However, there was an effect for diagnosis on the entropy of the Markov matrix for all orders $\left(0^{\text {th }}\right.$ : $\mathrm{F}=9.7, \mathrm{p}<0.01 ; 1^{\text {st }}: \mathrm{F}=12.3, \mathrm{p}<0.001 ;$ and $\left.2^{\text {nd }}: \mathrm{F}=6.3, \mathrm{p}<0.01\right)$ (see Fig. 2b for the characteristic effect for $2^{\text {nd }}$ order Markov, Table 4 for others), with the entropy of TD children universally lower than the entropy of ASD children. This suggests that there is less "exploration" of the non-stimulus - stimulus circuit by TD children, i.e. ASD children are making proportionally more transitions to and from non-stimulus states. It is important to note the difference between transition count and entropy calculations: the former is a raw tabulation which can be highly variable depending on the child; the latter is a relative measure which takes the context of the scanning circuit into account.

\section{B. Level 2 (Mid-Level): Face Saliency}

Here we consider the circuit spanning non-key and key features of the body of the stimulus. The key areas are further broken down into the eyes and mouth.

Analysis of looking times at the key features indicated a significant effect of diagnosis $(\mathrm{F}=4.3, \mathrm{p}<.05)$ (Fig. 3a), and a significant age $\mathrm{x}$ diagnosis interaction, $(\mathrm{F}=9.8, \mathrm{p}<.01)$. For the non-key area, there was only a significant age $\mathrm{x}$ diagnosis interaction, $(\mathrm{F}=6.6, \mathrm{p}<.05)$. A planned comparison revealed that this effect was driven partially by a significant increase in looking at both regions for TD (non-key features: $\mathrm{F}=4.3, \mathrm{p}<.05$; key features: $\mathrm{F}=.6 .4, \mathrm{p}<.05)$. These results taken together suggest that the amount devoted to key and non-key regions at age group 1 by both ASD and TD children is quite similar; similarly, ASD behaviors do not change for these regions 
between the two time points. However, there is a significant increase in looking at the face, and, in particular, at critical areas of the face, in TD children.

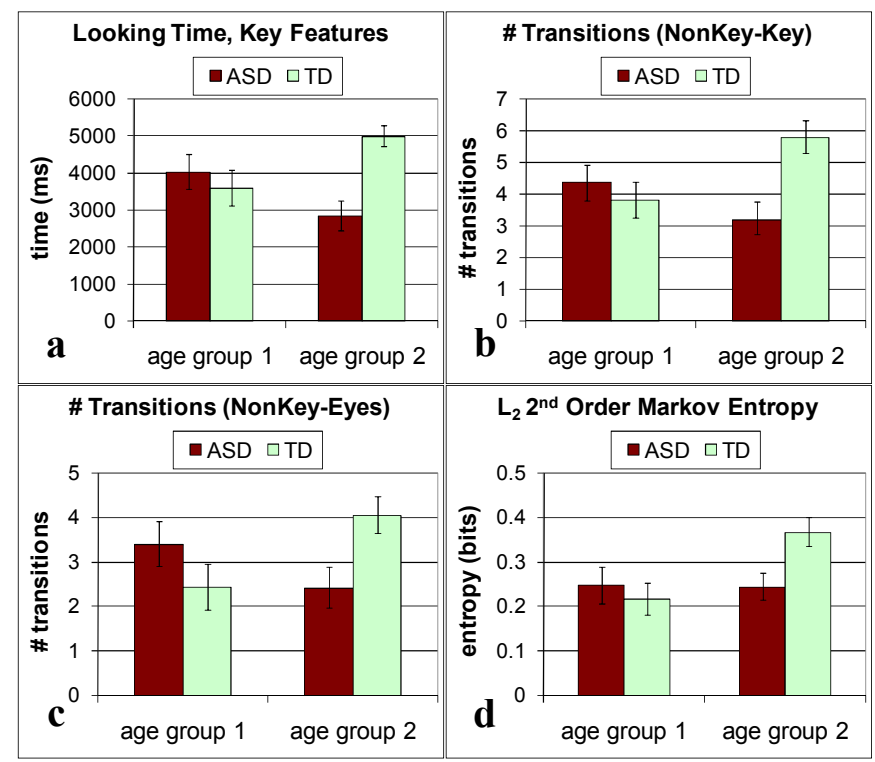

Figure 3. Measures from Level 2 circuit: (a) total average key features looking time (b) average number of non-key - key transitions per trial (c) average number of non-key - eyes transitions (b) Markov matrix entropy of key - mouth - eyes circuit.

We also compared the number of transitions between nonkey areas and the mouth and eyes areas (Fig. 3b). TD children transitioned between non-key areas and the mouth significantly more frequently than ASD children $(\mathrm{F}=4.1, \mathrm{p}<.05)$ (see Table 2 ). Analysis of the number of transitions between non-key areas and the eyes revealed only a significant age $\mathrm{x}$ diagnosis interaction $(\mathrm{F}=9.7, \mathrm{p}<.01)$ (Fig. 3c), suggesting an increase in frequency of shifts between non-key and eye areas in older TD children $(\mathrm{F}=7.3, \mathrm{p}<.05)$, but not in the ASD children.

For our Markov transitions, there was a main effect of both age and age $\mathrm{x}$ diagnosis on entropy for order two models (respectively $\mathrm{F}=4.5, \mathrm{p}<.05 ; \mathrm{F}=4.8, \mathrm{p}<.05$ ) with $\mathrm{TD}$ children having a greater entropy in age group 2 as compared to age group $1(\mathrm{~F}=9.7, \mathrm{p}<.01)$ (Fig. 3d) and no change in ASD. Again, this is consistent with the notion that TD children explore critical areas in a less deterministic fashion as they get older. By contrast, exploration measures in ASD do not differ between two years and four years of age.

\section{Level 1 (Ground Level): Canonical Scanning}

Here we consider the circuit spanning the mouth and eyes. The eyes can be divided into the left eye and the right eye.

A 2 (age) x 2 (diagnosis) analysis of looking time at the mouth area indicated a main effect of diagnosis $(\mathrm{F}=10.5$, $\mathrm{p}<.01$ ), with TD children looking at the mouth more than their ASD peers (Fig. 4a). An analogous analysis on the eye region indicated a significant age $\mathrm{x}$ diagnosis interaction $(\mathrm{F}=5.8$, $\mathrm{p}<.05$ ). This interaction was due to TD children, in age 2, looking at the eyes for longer periods than at age 1 (Fig. 4b).
There was no significant difference in looking time at the eyes between the two ASD groups.

For transitions, there was a significant effect of age on the number of transitions between the eyes and the mouth $(\mathrm{F}=4.9$, $\mathrm{p}<.05)$ as interaction $(\mathrm{F}=6.0, \mathrm{p}<.05)($ Fig. $4 \mathrm{c})$. Again, there was a significant increase in the number of transitions from age 1 to 2 in TD children $(\mathrm{F}=9.9, \mathrm{p}<.01)$, but not in the ASD groups. Additionally, there was an interaction of age $\mathrm{x}$ diagnosis on transition entropy for the full circuit of mouth-left-eye-right-eye $(\mathrm{F}=7.3, \mathrm{p}<.01)$ caused by an increase in entropy for TD children at age 2 as compared to age 1 .

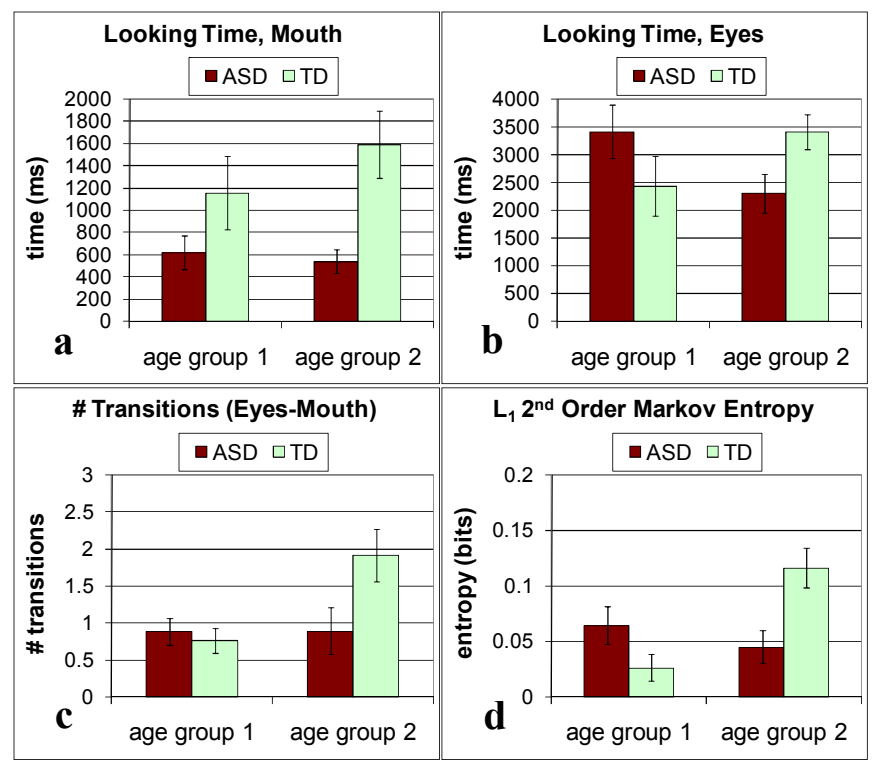

Figure 4. Measures from Level 1 circuit: (a) total average mouth looking time (b) eyes looking time (c) number of eyes-mouth transitions (b) Markov matrix entropy of mouth - left eye - right eye circuit.

For Markov analysis (Fig. 4d), we found a significant effect of age on entropy for both the $1^{\text {st }}$ order and $2^{\text {nd }}$ order chain ( $\mathrm{F}=6.27, \mathrm{p}<.05 ; \mathrm{F}=5.3, \mathrm{p}<.05$, respectively) as well as significant interaction effects $(\mathrm{F}=6.1, \mathrm{p}<.05 ; \mathrm{F}=12.3, \mathrm{p}<.001)$. There were no differences between older and younger ASD children for either $1^{\text {st }}$ or $2^{\text {nd }}$ order chains. However, there was a significant increase in entropy for both $1^{\text {st }}$ order and $2^{\text {nd }}$ order chains in TD controls $(\mathrm{F}=15.6, \mathrm{p}<.001 ; \mathrm{F}=17.3, \mathrm{p}<.001)$.

These results, taken together, suggest a functional aberration from typical development in canonical facial feature scanning in children with ASD. At a time when typical children seem to be gravitating towards focusing on core features such as the mouth and eyes, children with ASD are found to be unchanging in their strategies from age group 1 to age group 2 .

\section{DISCUSSION}

We return to our original question: how do the face scanning strategies of children with autism change in early childhood? In order to answer this question, we first look at how TD children change from two years of age to four years of age. At the top level, older TD children pay more attention to faces than younger TD children. This increasing attention 
trickles down to the next level, where increases in looking times at both key and non-key features are found. Again, at the bottom level, increases in looking times towards the eyes are found. All things being equal, we would expect to find a similar pattern in the number of transitions. This is exactly what we find: as TD children become older they begin to transition more often between non-key features and the eyes and the eyes and the mouth. By contrast, the number of transitions between non-stimulus and stimulus regions does not change, though the total time in stimulus regions increases. This pattern suggests that older TD children scan as frequently between the face and extraneous non-face regions as younger TD children, but that when they look at the face, they stay for longer and when they look away from the face, they stay for less, suggesting the development of either an increasing salience for faces, and, in particular, the eyes, or an increased ability to ignore distraction and irrelevant scene details.

Entropy results are not as dependent on the total number of transitions as they are on the balance of scanning between regions. At the bottom level, we find increasing exploration between eyes and mouth as TD children grow older, suggesting increased monitoring of the canonical face scanning circuit. Similarly, at the mid-level, we find a trend towards increased exploration of all areas of faces. Combined with the top-level finding of no changes in stimulus to non-stimulus scanning, the results suggest that increases in exploratory behavior in TD become more pronounced when zooming into the more inner circuits of face scanning. Thus, in TD children we see a very stable pattern of results, with attention ramping towards areas typically considered informative from a social and communicative point of view as the children age.

By contrast, at the top level, older ASD children look more at non-stimulus regions and less at stimulus regions than younger ASD children, suggesting a decreasing saliency for faces. This contrasts with ASD results for more inner regions, where no changes with age in looking times for key, non-key, mouth, or eyes were found. This implies that the trend of decreasing attention towards faces at the top level is not driven by any particular specific face region. Similarly no changes in age were found for transitions and entropy measures in ASD. This suggests that a possible answer to the question, "how do scanning strategies change in autism?" is: they don't. They don't increase looking at key features or the eyes and they don't increase their exploration of critical regions of the face.

The compounding influence of atypical exposure is a factor which may contribute to the differences we observe. It is useful to consider the effects found in the laboratory within the ecological scope of the affected child [12]. Given that we have found, at every level of our cascaded analysis, behaviors in autism deviating significantly from the typical trajectory, it is an open question as to how these deviations might shape the atypical social and cognitive environment. We note that decreased looking at particularly relevant facial features would imply a decreased experience for those features in that child's life. It is possible that this decreased experience depresses the typical development of configural or holistic face processing, leading to reduced stimulation in certain higher-level cortical social-affective circuitry, which in turn leads to decreased social motivation, which leads back to reduced experience.
In grounding these cascading effects, it is useful to consider the level one (ground level) circuit of the eyes and mouth for the younger age group. At this age, we find no differences between ASD and TD children in total number of transitions between regions or in entropy. However, we do find that TD children look more at the mouth than ASD children. One possible explanation for this effect is that TD children are making as frequent transitions between regions as ASD children, but when they encounter the mouth, they stay for a longer period of time. This mouth salience hypothesis could be driven in part by differences observed in preferences for elementary features. For example, typical children at two years of age might attend to the mouth in static images, having built up an expectation for novel motion from this area. By contrast, in ASD, a preference for high areas of contrast combined with decreased sensitivity for motion [13] might bias the child in ASD towards looking predominantly at unique high contrast areas (e.g. the boundary between the sclera and the face or pupil) and less towards the mouth.

It is also possible that trial-level effects are confounding our interpretation of our summary statistics, which are assumed to be valid at a micro-level of analysis. For example, it is possible that both ASD children and TD children attend to the initial area they look upon for a long period of time, and then saccade away to another area. It may be the case that ASD children are more likely to initially focus upon the eyes, and that TD children are more variable in their choice of initial fixation. In this case, the increased variation of TD children could be viewed as noise. Interestingly, this "noise" would increase the chances that interesting holistic aspects of faces could be uncovered. This interpretation and the perceptual interpretation are not mutually exclusive.

We should note that the trend of older TD children looking longer at the mouth than ASD children is a result which might be considered unexpected given prior results in dynamic scenes by Klin et al. [4]. There are several possibilities which might account for this difference, however. First, the switch from a static face scene to a dynamic social interaction scene is a huge leap in cognitive load, social affective circuitry, and basic perceptual saliency. Second, the individuals in Klin et al.'s study were higher functioning individuals with autism; the individuals in this study were more impaired, having a nonverbal mental age developmental quotient of 80 and a verbal mental age developmental quotient of 61 [7]. Third, it is possible that the developmental effects driving mouth-looking simply haven't come into fruition at the ages examined in this work. For example, if the learning of language interacts with looking at the mouth in autism (for example in aiding phoneme recognition), then it is possible that individuals who are delayed in the use of language would develop compensatory mechanisms at a much later than four years of age.

Methodologically, it has been our goal to use measures that apply the least amount of data manipulation to the eye-tracking stream as possible. There are several reasons for this. First, nothing is as comparable, or as easy, as doing nothing to your data. Second, the differential loss from complex eye-tracking measures is something that has been little examined; however, our experience is that commonly used eye-tracking tools, such as fixation analysis, routinely allow one to shape one's data in 
an arbitrary and invisible manner $[14,15]$. Nonetheless, the comparison of our results to standard fixation measures is a necessary avenue to be explored.

There have been several simple methods proposed that use variations on entropy as a measure in eye-tracking. For example, Kruizinga et al. [16] employ an entropy measure on a single row of the transition matrix to calculate the entropy of specific ROIs. Althoff and Cohen [17] combine the entropy of matrix cells with row and column entropy totals, normalized by the column entropy total, to obtain a measure they term S1 and S2. Our method proceeds similarly and is quite simple, as it simply reflects the entropy rate of a discrete Markov chain [11]. We have seen that this measure is sufficient to generate natural results that are quite interpretable. As it is derived from fundamental information theory, there is also a rich basis for expanding its application.

Our measures work, in part, because we have broken down our investigation into several easy-to-digest pieces. For example, our $2^{\text {nd }}$-order Markov entropy measure can span a single region which is being saccaded over since it has a history of two prior states. As there are at most three regions under consideration at any point in our analysis, this measure is particularly appropriate. This leads us to an important point: the measures employed for analysis should be matched with the appropriate simplifying solutions.

Several limitations of this study need to be noted. For one, the design of our trials is cross-sectional and thus we cannot be sure that the age groups are completely comparable. Similarly, selection criteria for valid trials were chosen quite arbitrarily and we have not measured any aspects of drop-out.

We also did not include a mental-age matched sample for our ASD population (e.g. a developmentally delayed group). Thus, the results we have found could be attributed to differences in cognitive functioning, though the interactions found would be more difficult to explain in this manner.

It is important to note that many trends apparent in the graphs, such as decreased looking times at eyes in older children with ASD, were not significant. It is possible that with a larger sample size these trends could become more prominent, changing the interpretation of our results.

The use of two different screen sizes for the older and younger populations is also a potential confound. We note, however, that within age group comparisons do not suffer from this deficit and that TD and ASD individuals typically do not behave in the same direction (i.e. both up or both down at time 2 from time 1). Also, our preliminary analysis has indicated no significant effect of the monitor size on scanning patterns.

Finally, our measures are fairly new and we have only begun to examine their limitations, interpretations, and interrelationships. However, we are reassured in part by the simple nature of many of our measures. Future work will consider technical issues such as the effect of data loss and sampling as well as methodological issues such as the applicability of our measures to other questions and domains.

\section{CONCLUSION}

We have presented a series of results which have shown some interesting facets regarding the scanning patterns of children with autism. We have also discussed some new avenues for obtaining measures from eye-tracking data, and offered some recommendations for a simple experimental methodology for analysis.

\section{ACKNOWLEDGMENT}

The authors would like to thank Gordon Ramsay, Summer Han, and Joseph Chang, as well as three anonymous reviewers, for their useful advice regarding this work.

\section{REFERENCES}

[1] American Psychiatric Society, Diagnostic and statistical manual of mental disorders, Washington, DC: 1994.

[2] Joseph R. M and Tanaka J, "Holistic and part-based face recognition in children with autism," Journal of Child Psychology and Psychiatry, vol. 44, May. 2003, pp. 529-542.

[3] J. Boucher and V. Lewis, "Unfamiliar face recognition in relatively able autistic children," Journal of Child Psychology and Psychiatry, vol. 33, 1992, pp. 843-859.

[4] A. Klin et al., "Visual Fixation Patterns During Viewing of Naturalistic Social Situations as Predictors of Social Competence in Individuals With Autism," Archives of General Psychiatry, vol. 59, 2002, p. 809.

[5] K.A. Pelphrey et al., "Visual Scanning of Faces in Autism," Journal of Autism and Developmental Disorders, vol. 32, 2002, pp. 249-261.

[6] C. Lord, Autism Diagnostic Observation Schedule: ADOS: Manual, Western Psychological Services, 2002.

[7] E.M. Mullen, "Mullen Scales of Early Learning," Circle Pines, MN: American Guidance Service, 1995.

[8] K. Chawarska et al., "Autism spectrum disorder in the second year: stability and change in syndrome expression," Journal of Child Psychology and Psychiatry, vol. 48, 2007, pp. 128-138.

[9] D. Lundqvist, A. Flykt, and A. Ohman, "The Karolinska Directed Emotional Faces," Available from the Department of Neurosciences, Karolinska Hospital, Stockholm, Sweden, 1998.

[10] R.L. Fantz, "Visual Experience in Infants: Decreased Attention to Familiar Patterns Relative to Novel Ones," Science, vol. 146, Oct. 1964, pp. 668-670.

[11] T.M. Cover and J.A. Thomas, Elements of Information Theory, WileyInterscience New York, 2006.

[12] A. Klin et al., "The Enactive Mind, or from Actions to Cognition: Lessons from Autism," Philosophical Transactions: Biological Sciences, vol. 358, Feb. 2003, pp. 345-360.

[13] F. Shic et al., "Measuring context: The gaze patterns of children with autism evaluated from the bottom-up," Development and Learning, 2007. ICDL IEEE 6th International Conference on, 2007, pp. 70-75.

[14] F. Shic, K. Chawarska, and B. Scassellati, "The incomplete fixation measure," Proceedings of the 2008 symposium on Eye tracking research \& applications, 2008, pp. 111-114.

[15] F. Shic, K. Chawarska, and B. Scassellati, "The Amorphous Fixation Measure Revisited: with Applications to Autism," 30th Annual Meeting of the Cognitive Science Society, Washington, DC: 2008.

[16] A. Kruizinga, B. Mulder, and D. de Waard, "Eye scan patterns in a simulated ambulance dispatcher's task," Developments in Human Factors in Transportation, Design, and Evaluation, D. de Waard, K. Brookhuis, and A. Toffetti, eds., Orbassano, Italy: Shaker Publishing, 2006, pp. 305-317.

[17] R. Althoff et al., "Eye Movement-based Memory Assessment," Current Oculomotor Research: Physiological and Psychological Aspects, 1999. 